\title{
Enterobacter sakazakii invades brain capillary endothelial cells, persists in human macrophages influencing cytokine secretion and induces severe brain pathology in the neonatal rat
}

Correspondence

Stacy M. Townsend

stacy.townsend@hotmail.com

Received 26 April 2007

Revised 12 June 2007

Accepted 29 June 2007

\author{
Stacy M. Townsend, ${ }^{1}$ Edward Hurrell, ${ }^{1}$ Ignacio Gonzalez-Gomez, ${ }^{2,3}$ \\ James Lowe, ${ }^{4}$ Jonathan G. Frye, ${ }^{5}$ Stephen Forsythe ${ }^{1}$ \\ and Julie L. Badger ${ }^{2,3}$
}

\author{
'School of Biomedical and Natural Sciences, Nottingham Trent University, Clifton Lane, Nottingham \\ NG11 8NS, UK \\ ${ }^{2}$ Department of Pathology, Children's Hospital Los Angeles, CA 90027, USA \\ ${ }^{3}$ University of Southern California Keck School of Medicine Los Angeles, CA 90027, USA \\ ${ }^{4}$ Department of Neurology, Queen's Medical Centre NHS Trust, Nottingham NG7 2UH, UK \\ ${ }^{5}$ Bacterial Epidemiology and Antimicrobial Resistance Research Unit, USDA, Agricultural Research \\ Service, 950 College Station Road, Athens, GA 30605, USA
}

\section{INTRODUCTION}

Enterobacter sakazakii is a rare cause of neonatal Gramnegative sepsis and severe disease manifestations such as meningitis and brain abscess formation. There is also a strong association between some cases of necrotizing enterocolitis and the colonization of Ent. sakazakii. The contamination of powdered infant formula with Ent. sakazakii has been linked to neonatal outbreaks resulting in devastating morbidity and death (van Acker et al., 2001; Biering et al., 1989; Coignard et al., 2006; Himelright et al., 2002; Jarvis, 2005; Muytjens et al., 1983). This has generated significant concern over the non-sterile condition of

Abbreviations: BBB, blood-brain barrier; CSF, cerebrospinal fluid; H\&E, haematoxylin/eosin; i.c., intracranially; i.p., intraperitoneally; PMA, phorbol 12-myristate 13-acetate; SOD, superoxide dismutase. this product and the regulatory standards governing the presence of this ubiquitous, opportunistic pathogen in infant formula powder (FAO-WHO, 2006). While recent studies have focused on the identification and classification of this genetically diverse species, very little is known concerning virulence (Drudy et al., 2006; Iversen et al., 2006; Iversen \& Forsythe, 2007). Studies on toxin production by Pagotto et al. (2003) showed the production of cytotoxins or enterotoxins by clinical isolates and therefore these may contribute to a possible mechanism of pathogenesis. There is considerable diversity within the Ent. sakazakii species, which can be divided into four cluster groups according to $16 \mathrm{~S}$ rDNA analysis (Iversen et al., 2004). It is of interest whether the virulence of Ent. sakazakii varies according to cluster group. The presence of lipopolysaccharide (LPS) in powdered infant formula has 
also been implicated in increasing the permeability of tissue barriers to intestinal bacteria including Ent. sakazakii (Townsend et al., 2007). Studies to determine the ability of Ent. sakazakii to penetrate the blood brain barrier (BBB) and cause meningitis are hindered due to lack of an animal model. Citrobacter spp. and Escherichia coli K1 elicit haematogenous meningitis and brain abscess development in the neonatal rat (Kim et al., 1997; Kline et al., 1988); however, similar dissemination is not observed with Ent. sakazakii and in vivo models of this infection may require the use of immunodeficient animal strains (Pagotto et al., 2007).

This study utilized intracranial inoculation of neonatal rats with Ent. sakazakii to facilitate histological characterization of the inflammatory response in vivo. Ent. sakazakii strains from cluster groups 1 and 2 were shown to significantly invade rat brain capillary endothelial cells (rBCEC4). Gene probing suggests that Ent. sakazakii survival in human (U937) macrophages in vitro may be influenced by sodA. In addition, we suggest that Ent. sakazakii may bias early IL10/IL-12 cytokine expression by macrophages, contributing to the exacerbation of disease.

\section{METHODS}

Bacterial strains and culture conditions. Ent. sakazakii strains representing each $16 \mathrm{~S}$ cluster group were used in this study (Table 1). The cluster 1 strain NTU658 (ATCC BAA-894) is currently being sequenced by Washington University in St Louis Genome Sequencing
Center (http://www.genome.wustl.edu). Citrobacter koseri SMT319 (Townsend et al., 2003), Citrobacter freundii NTU340, E. coli K1 meningitic cerebrospinal fluid (CSF) isolate RS218 (O18:K1:H7) and Salmonella enterica serovar Enteritidis (NCTC 3046) were used as positive controls and non-pathogenic E. coli K-12 served as a negative control. For animal studies, gentamicin protection assays and cytokine secretion assays, bacteria were aerobically grown at $37{ }^{\circ} \mathrm{C}$ to mid-exponential phase in brain heart infusion (BHI) broth and washed twice with and resuspended in cold PBS. Concentrations of bacterial inoculations were determined by $\mathrm{OD}_{600}$ and confirmed by plate count enumeration.

Serum complement resistance and acid tolerance. Serum sensitivity and acid tolerance were assessed via turbidimetric analysis and confirmed by viable plate counts. BHI broth cultures were grown with shaking to exponential phase and resuspended to obtain $10^{7}$ cells $\mathrm{ml}^{-1}$. E. coli $\mathrm{K}-12$ was used as a negative control. For serum studies over $2 \mathrm{~h}, 50 \mu \mathrm{l}$ bacterial suspension was added to $50 \mu \mathrm{l}$ non-immune or heat-inactivated $\left(56{ }^{\circ} \mathrm{C}\right.$ for $30 \mathrm{~min}$ ) human pooled serum (HPS) in triplicate. For acid tolerance studies over 6 h, $100 \mu \mathrm{LB}$ broth $\mathrm{pH}$ adjusted to 4 and buffered with citrate/phosphate buffer was added to $50 \mu \mathrm{l}$ of culture in triplicate. The mixtures were incubated at $37{ }^{\circ} \mathrm{C}$ and the $\mathrm{OD}_{600}$ was determined at $30 \mathrm{~min}$ intervals. Percentage survival was determined as (Number of bacteria that survived $2 \mathrm{~h}$ treatment/Inoculum size $) \times 100$. Strains able to survive in HPS at levels equal to or greater than that observed initially were considered resistant.

Superoxide dismutase (SOD) activity. SOD activity in Ent. sakazakii cell lysates was evaluated using the Superoxide Dismutase Assay kit II (Calbiochem). Yersinia enterocolitica strain 8081 served as a positive control (Roggenkamp et al., 1997). Bacterial pellets collected from $2 \mathrm{ml}$ overnight cultures were sonicated in ice-cold $20 \mathrm{mM}$ HEPES buffer containing $1 \mathrm{mM}$ EGTA, $210 \mathrm{mM}$ mannitol

Table 1. Summary of strains used in this study

\begin{tabular}{|c|c|c|c|c|}
\hline NTU strain ID & Source $\dagger$ & Origin & $\begin{array}{l}\text { 16S rDNA cluster } \\
\text { group }\end{array}$ & Organism \\
\hline 1 & NCTC $11467^{\mathrm{T}}$ & Child's throat & 1 & Ent. sakazakii \\
\hline 2 & ATCC 12868 & Unknown & 1 & Ent. sakazakii \\
\hline 658 & ATCC BAA-894 & Infant formula & 1 & Ent. sakazakii \\
\hline 57 & NTU & Milk powder & 2 & Ent. sakazakii \\
\hline 3 & ATCC 51329 & Unknown & 3 & Ent. sakazakii \\
\hline $16^{*}$ & NTU & Herb & 3 & Ent. sakazakii \\
\hline $530^{*}$ & HMMP13 & Milk powder & 3 & Ent. sakazakii \\
\hline $566^{*}$ & CDC-3523-75 & Bone marrow & 3 & Ent. sakazakii \\
\hline 84 & NTU & Herb & 4 & Ent. sakazakii \\
\hline $564^{*}$ & CDC-5960-70 & Blood & 4 & Ent. sakazakii \\
\hline $582^{*}$ & NCTC 9844 & Unknown & 4 & Ent. sakazakii \\
\hline $583^{*}$ & NCTC 9846 & Unknown & 4 & Ent. sakazakii \\
\hline NA & NCTC 3046 & Unknown & NA & Salmonella Enterica \\
\hline NA & CHLA & CSF & NA & E. coli $\mathrm{K} 1 \mathrm{RS} 218$ \\
\hline $\mathrm{NA}$ & CHLA & Unknown & NA & E. coli K-12 HB101 \\
\hline NA & WU & CSF & NA & C. koseri SMT319 \\
\hline 340 & NTU & Salad & NA & C. freundii \\
\hline
\end{tabular}

NA, Not applicable.

${ }^{*}$ Used in PCR studies only.

$\dagger^{\mathrm{T}}$, Type strain; NTU, Nottingham Trent University; CHLA, Children's Hospital Los Angeles; WU,

Washington University Medical School (Joseph St Geme III). 
and $70 \mathrm{mM}$ sucrose ( $\mathrm{pH} 7.2)$. Centrifugation at $1500 \mathrm{~g}$ for $5 \mathrm{~min}$ at $4{ }^{\circ} \mathrm{C}$ cleared the supernatants used in the assay to detect superoxide radicals according to the manufacturer's instructions. Two independent assays were performed in duplicate.

Tissue culture cell line cultivation and invasion assays. U937 macrophages were obtained from the ATCC (CRL-1593.2) and seeded into $75 \mathrm{ml}$ tissue culture flasks (Sundstrom \& Nilsson, 1976). Cells were cultivated, activated and plated as described previously (Townsend et al., 2003). Cells were gently washed with RPMI to remove residual phorbol 12-myristate 13 -acetate (PMA) following activation, and fresh medium was added prior to inoculation with unopsonized bacteria. U937 human macrophages were infected at an m.o.i. of 10 for $45 \mathrm{~min}$ at $37{ }^{\circ} \mathrm{C}$ in $5 \% \mathrm{CO}_{2}$. After a $45 \mathrm{~min}$ incubation period, the medium was aspirated and replaced with U937 macrophage medium supplemented with $100 \mu \mathrm{g}$ gentamicin $\mathrm{ml}^{-1}$ and incubated for an additional $45 \mathrm{~min}$ at $37{ }^{\circ} \mathrm{C}$ in $5 \% \mathrm{CO}_{2}$. Macrophages were washed twice, lysed with $0.5 \%$ Triton X, serially diluted, and plated to determine the number of intracellular bacteria at various time points (Zaidi et al., 1996). The viability of the bacterial strains tested was not affected by $0.5 \%$ Triton X treatment. For persistence assays, cells were replenished daily with fresh medium containing $10 \mu \mathrm{g}$ gentamicin $\mathrm{ml}^{-1}$ (above the MIC). Trypan Blue exclusion staining indicated that macrophage viability ranged from 80 to $95 \%$ and was maintained for at least $96 \mathrm{~h}$. For persistence assays, results for each time point are presented as the percentage of inoculum that was intracellular. All assays were performed in triplicate at least twice.

The rat brain capillary endothelial cell line (rBCEC4) was a kind gift from I. E. Blasig (Forschungsinstitut für Molekulare Pharmakologie, Berlin, Germany). Following the 22nd subculture rBCEC4 cells were seeded at $1 \times 10^{5}$ cells per well into collagen-coated 24 -well plates and left to adhere for $48 \mathrm{~h}$. The medium contained DMEM, $4.5 \mathrm{~g}$ glucose $\mathrm{l}^{-1}, 1.2 \mathrm{mM}$ glutamine, $100 \mathrm{U}$ penicillin $\mathrm{ml}^{-1}, 100 \mu \mathrm{g}$ streptomycin $\mathrm{ml}^{-1}, 2.5 \mu \mathrm{g}$ amphotericin B ml ${ }^{-1}$ (Sigma-Aldrich), $100 \mu \mathrm{g}$ heparin $\mathrm{ml}^{-1}, 110 \mu \mathrm{g}$ sodium pyruvate $\mathrm{ml}^{-1}$ (Sigma), $10 \mu \mathrm{g}$ ECGF $\mathrm{ml}^{-1}$ (endothelial growth factor; Axxora), $10 \%$ fetal bovine serum (Blasig et al., 2001). Each bacterial strain was grown in brain heart infusion broth (BHI; CM 10322, Oxoid) to mid-exponential phase from overnight cultures and inoculated in triplicate at an m.o.i. of $1: 100$. Inoculated cells were incubated with $5 \% \mathrm{CO}_{2}$ at $37{ }^{\circ} \mathrm{C}$ for $1.5 \mathrm{~h}$ as previously described (Badger et al., 1999). In order to quantify bacterial invasion $100 \mu \mathrm{g}$ gentamicin $\mathrm{ml}^{-1}$ was added to each well and incubated for $30 \mathrm{~min}$. Then the cells were washed twice with PBS and treated with trypsin to dislodge the adherent cells. The rBCEC4 cells were lysed with $0.5 \%$ Triton $\mathrm{X}$ and serial dilutions were plated on nutrient agar. Cell integrity following invasion was qualitatively assessed using Trypan Blue staining after $2 \mathrm{~h}$ incubation. E. coli K-12 and C. koseri SMT319 were used as negative and positive controls, respectively. Data are presented as percentage invasion as determined by (Number of bacteria recovered/Number of bacteria inoculated) $\times 100$.

Animal studies. Timed-pregnant (E14) Sprague-Dawley rats (Charles River Laboratories) were obtained and gave birth in our vivarium after a 21 day gestation period. Litters averaged 12 pups and were kept with their mother in an opaque polypropylene cage under a Small Animal Isolator (Forma Scientific). Two- to three-day-old rat pups were anaesthetized with isoflurane and inoculated. For serum cytokine studies (described below), $10^{7}$ c.f.u. in $0.1 \mathrm{ml}$ PBS were inoculated intraperitoneally (i.p.). For histological studies, $10^{3}$ c.f.u. in $0.002 \mathrm{ml}$ PBS were inoculated intracranially (i.c.); i.c. inoculations were administered through a burr hole produced by a 26-gauge needle at coordinates approximately $5 \mathrm{~mm}$ caudal to the right eye and $2 \mathrm{~mm}$ right of the sagittal suture. A 33-gauge, single internal cannula (Plastics One) was attached to a Hamilton 1801RN $10 \mu \mathrm{l}$ syringe (Hamilton Company) with a 22-gauge needle via 24-gauge standard wall tubing, to administer the dose. The needle was inserted perpendicular into the right parietal area approximately $2 \mathrm{~mm}$ deep from the external surface; $2 \mu \mathrm{l}$ was injected over 1 min then the needle was carefully retracted. For the study reported in Table 2, rat pups were anaesthetized as they succumbed to infection up to 9 days post i.c. inoculation. Blood samples were aseptically collected via intracardiac puncture, as previously described (Badger \& Kim, 1998). Blood samples $(10 \mu \mathrm{l})$ were inoculated in LB and plated on agar plates. Rats were euthanized and whole brains were removed for histological analysis. All animal experiments were performed according to protocols approved by CHLA Institutional Animal Care and Use Committee (IACUC).

Histopathology. Whole neonatal rat brains were fixed in $10 \%$ buffered formalin, routinely processed, then paraffin embedded. Coronal sections of $4-5 \mu \mathrm{m}$ were cut and stained with haematoxylin and eosin (H\&E). Histological analysis of the midbrain was completed by one blinded pathologist (J. L.)

Electron microscopy. Transmission electron microscopy was used to visualize Ent. sakazakii within rat macrophages. Ent. sakazakiiinfected rat brain samples were fixed with $2.5 \%$ glutaraldehyde in $0.1 \mathrm{M}$ PBS. Samples were post-fixed for $1 \mathrm{~h}$ with $2 \% \mathrm{OsO}_{4}$, rinsed, dehydrated through graded ethanol solutions, and embedded in polypropylene oxide. Ultrathin sections mounted on collodion grids (single hole) were stained with uranyl acetate and lead citrate and examined with a Philips CM transmission electron microscope.

DNA isolation, gene identification and PCR. Genomic DNA was prepared from $1.5 \mathrm{ml}$ overnight culture grown in LB broth using a GenElute Bacterial Genomic DNA kit (Sigma) according to the manufacturer's instructions. DNA-DNA microarray hybridization of Ent. sakazakii DNA to Salmonella enterica serotype Typhi and Typhimurium virulence gene arrays (Porwollik et al., 2003) identified homologues to Ent. sakazakii genes sodA and ompA related to macrophage survival and serum resistance. The Ent. sakazakii genome project at Washington University School of Medicine Genome Sequencing Center (http://www.genome.wustl.edu) provided sequence information of the Ent. sakazakii gene homologous to sodA and $\operatorname{sodB}$ from $S$. Typhimurium and PCR primers were designed to probe the chromosomal DNA. The PCR reaction mixture contained $5 \times$ Green GoTaq Flexi Buffer (Promega), $2.5 \mathrm{mM}$ $\mathrm{MgCl}_{2}, \quad 0.2 \mathrm{mM}$ each dNTP, $0.5 \mu \mathrm{M}$ SodA1-F (5'-GTCAAC AACGCTAACG-3') and SodA1-R (5'-CCCATCAGCGGGGAAT-3') primers, $2.5 \mathrm{U}$ GoTaq Flexi DNA polymerase (Promega), $100 \mathrm{ng}$ template DNA, and nuclease-free water to a final volume of $50 \mu \mathrm{l}$. The thermocycler (Genius FGEN 05 TD; Techne) was programmed as follows. An initial denaturing step at $94{ }^{\circ} \mathrm{C}$ for $2 \mathrm{~min}$ was followed by 30 cycles of denaturation at $94{ }^{\circ} \mathrm{C}$ for $30 \mathrm{~s}$, annealing at $55{ }^{\circ} \mathrm{C}$ for $1 \mathrm{~min}$ and extension at $72{ }^{\circ} \mathrm{C}$ for $1 \mathrm{~min}$, and a final extension step of $72{ }^{\circ} \mathrm{C}$ for $5 \mathrm{~min}$; this yielded a $352 \mathrm{bp}$ product. The ompA gene has recently been identified and was amplified using primers ESSF and ESSR, which yield a 469 bp product following PCR conditions described previously (Mohan Nair \& Venkitanarayanan, 2006). PCR products were visualized on $1 \%$ agarose gels stained with $0.5 \mu \mathrm{g}$ ethidium bromide $\mathrm{ml}^{-1}$.

Quantitative analysis of cytokine secretion. ELISA was used to quantify cytokine secretion (IL-10, IL-12 and TNF $\alpha$ ) from U937 macrophages following Ent. sakazakii uptake. Human IL-10 (sensitivity $<1 \mathrm{pg} \mathrm{IL}-10 \mathrm{ml}^{-1}$ ), IL-12 (sensitivity $<0.2 \mathrm{pg} \mathrm{IL}-12 \mathrm{ml}^{-1}$ ), and $\mathrm{TNF} \alpha$ (sensitivity $<1.7 \mathrm{pg} \mathrm{TNF} \alpha \mathrm{ml}^{-1}$ ) ELISA kits (Biosource International) were used to measure cytokines secreted into the macrophage supernatant at 6 and $24 \mathrm{~h}$ post-inoculation with each strain in this study. E. coli O111:B4 LPS or PMA (Sigma) was used as? a positive control. ELISA was also used to measure rat pup IL-10 (sensitivity $<5$ pg IL-10 $\mathrm{ml}^{-1}$ ) concentrations (Biosource 
International). Rat serum was isolated $24 \mathrm{~h}$ after i.p. injection of Ent. sakazakii strain NTU2 or NTU658 and IL-10 concentration was measured. In accordance with the manufacturer's instructions, each sample (serum was diluted 1:10) was run in duplicate wells and the absorbance values were averaged. Standard curves were generated using the cytokine standards supplied by the manufacturer.

\section{RESULTS AND DISCUSSION}

\section{Histological characterization of rat brain inflammatory response following intracranial Ent. sakazakii infection}

Ent. sakazakii causes opportunistic infections and is reported to be associated with the consumption of infant formula in neonates. Traversing the gut and entering the brain requires invasive capabilities and immune evasion techniques that are yet to be fully elucidated. In this study, genetically distinct Ent. sakazakii strains were selected and tested in vivo and in vitro to better characterize mechanisms of virulence and the host immune response to infection. Ent. sakazakii strains from cluster 1 are most commonly isolated from food, clinical and environmental sources and have been associated with clinical disease. Cluster groups 2, 3, and 4 are dissimilar and less commonly isolated yet these strains are still recovered from clinical sources. The pathogenic potential of these strains has not been previously described.

For the comparative study summarized in Table 2, strains from clusters 1 and 2 were inoculated into rat pups via intracranial injection. Blood and brains were collected and examined between 6 and 9 days post-inoculation to observe evidence of sepsis and chronic-pattern inflammation. This study showed that NTU1 was attenuated in comparison to other strains such as NTU2, which initiated chronic-pattern inflammation (lymphocytic predominance) in $83 \%$ of rats tested (Table 2). This provides evidence that even within genetically similar strains there are significant differences in the host immune response. These differences may be attributed to distinctions at the level of protein expression, although it cannot be ruled out that they may be due to extensive subculturing resulting in a loss of virulence in NTU1. Strain NTU658, from cluster group 1, is reported as having caused a neonatal outbreak and its genome is currently being sequenced. This strain

Table 2. Comparative in vivo Ent. sakazakii study

\begin{tabular}{|llcrc|}
\hline Strain & \multicolumn{1}{c}{ Origin } & $\begin{array}{c}\text { Cluster } \\
\text { group }\end{array}$ & Meningitis & $\begin{array}{c}\text { Chronic-pattern } \\
\text { inflammation }\end{array}$ \\
\hline NTU1 & Child's throat & 1 & $0 \%(0 / 7)$ & $0 \%(0 / 7)$ \\
NTU2 & Unknown & 1 & $83 \%(5 / 6)$ & $83 \%(5 / 6)$ \\
NTU658 & Milk powder & 1 & $33 \%(2 / 6)$ & $33 \%(2 / 6)$ \\
NTU57 & Milk powder & 2 & $50 \%(3 / 6)$ & $33 \%(2 / 6)$ \\
\hline
\end{tabular}

caused meningitis and chronic-pattern inflammation in $33 \%$ of infected rat pups (Table 2). Strain NTU57 (cluster group 2) also caused chronic-pattern inflammation in $33 \%$ of infected rat pups, and $50 \%$ of the rat pups developed meningitis as defined by inflammation within the meninges following histological analysis (Table 2). No bacterial growth was detected in blood samples; thus no evidence of sepsis was obtained. This comparative study identified strain NTU2 as having the highest occurrence of chronicpattern inflammation, and this strain was used for further kinetic studies.

Intracranial injection of Ent. sakazakii was performed in a kinetic study to describe the progression of brain inflammation. The injection site was located in the right cerebral cortex at the approximate junction of the forebrain and midbrain at a depth $(2 \mathrm{~mm})$ not penetrating the ventricle. Rat pups were sacrificed at various time points up to 9 days. Histological analysis of H\&E-stained coronal sections elucidated the inflammatory response. Fig. 1(a) is an example of normal brain histology observed in rat pups 9 days post-infection with NTU1 (most indistinguishable from controls) to compare to the severe inflammatory reaction observed in NTU2-infected rat pups. Only three rat pups inoculated with NTU1 had extremely small foci of inflammatory cells (mostly neutrophils) in the cingular gyrus, near the base of the brain, or near the meninges. Rat pups inoculated with NTU2 had severe bilateral ventriculitis, meningitis and marginalized neutrophils in local blood vessels 3 days post-inoculation (Fig. 1b, c). Lymphocytes and microglia were activated, reactive astrocytes were observed and inflammation was associated with a micro-haemorrhage (Fig. 1d). The occurrence of oedema and flocculation suggests that increased vascular permeability and fibrin liberated from blood vessels may impair drainage of CSF, causing hydrocephalus. The choroid plexus also harboured inflammatory cells and secreted fibrin, which was not prevalent in the C. koseri model (Townsend et al., 2003). Leptomeningitis, ventriculitis and ventriculomegaly, with massive dilation of the ventricles, worsened 6 days post-inoculation (Fig. 1e). Morphological evidence of neuronal or glial cell death via apoptosis was suggested by hyperchromatic, condensed nuclei within the cortex (Fig. 1f) and could be induced by bacterial factors such as LPS or other secreted endotoxins (Pagotto et al., 2003). Further, neuronal death and cell lyses releases acid hydrolase and causes liquefaction and necrosis of brain tissue. It follows that there is also evidence of ischaemic damage (possibly due to oedema and pressure), reduced neuronal density and liquefaction within the cortex; these factors could also influence the observed neuronal cell death. Neutrophils were still marginated and infiltrating into the cortex with other inflammatory cells, causing multiple foci of cerebritis and small ischaemic lesions more than a week after inoculation (Fig. 1g, h, i).

The multiple inflammatory foci provide evidence that the bacteria can enter the CSF circulation. The glial limitans, normally a good barrier preventing the extension of 

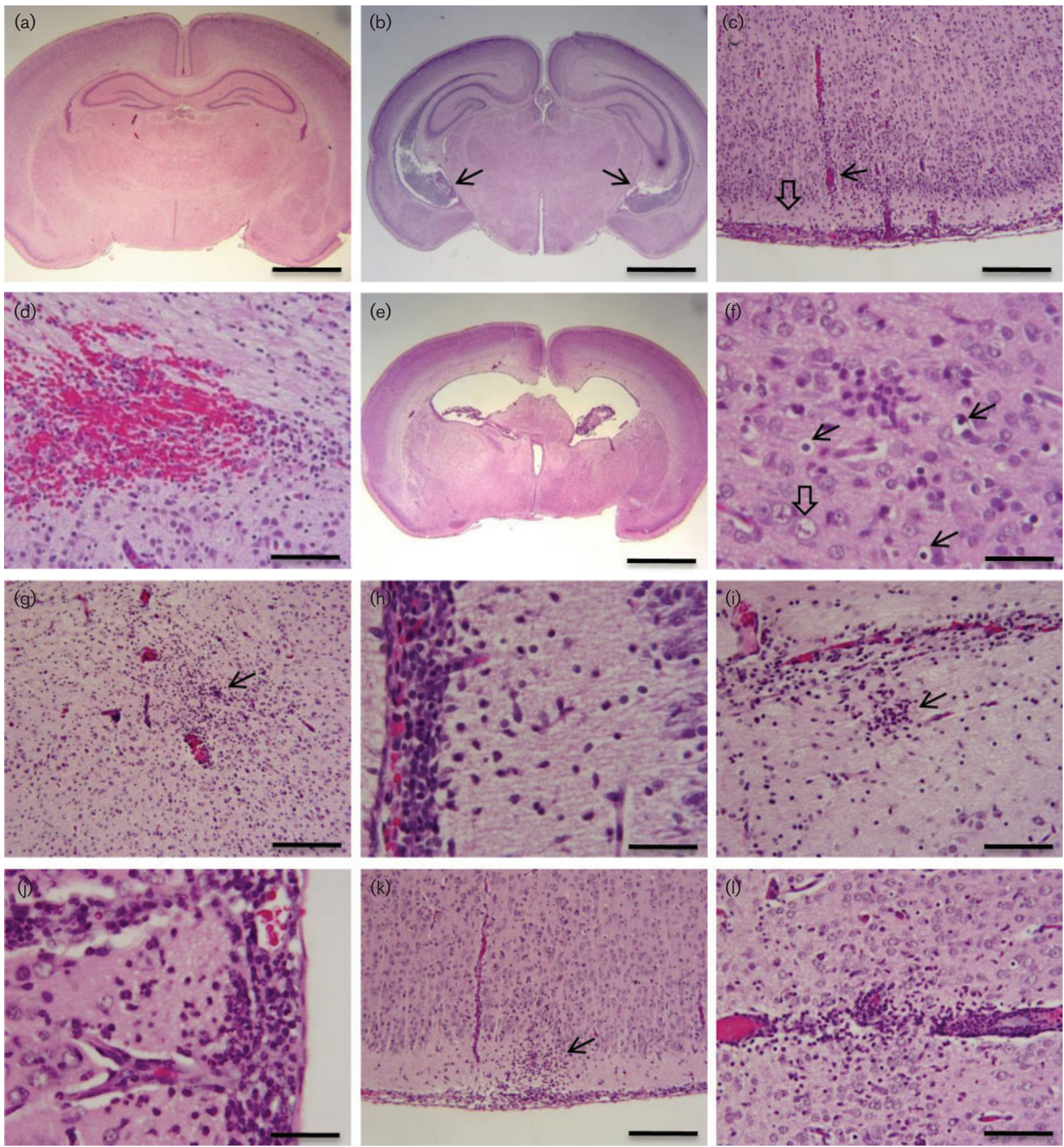

Fig. 1. Histological analysis of Ent. sakazakii in the infant rat brain. Ent. sakazakii NTU2 was inoculated into rat pups via i.c. injection. Rat pups were sacrificed at various time points after inoculation. Histological analysis of H\&E-stained coronal sections elucidated the inflammatory response. Bars: $2 \mathrm{~mm}$ (a, b, e), $500 \mu \mathrm{m}(\mathrm{k}), 200 \mu \mathrm{m}(\mathrm{c}, \mathrm{g}), 100 \mu \mathrm{m}$ (d, i, l) and $50 \mu \mathrm{m}$ (f, h, j). (a) Rat pup inoculated with Ent. sakazakii NTU1 with normal brain histology. (b) Rat pup inoculated with Ent. sakazakii strain NTU2 showing severe bilateral ventriculitis 3 days post-inoculation. (c) Marginalization of neutrophils (solid arrow) and leptomeningeal inflammation (open arrow) at day 3. (d) Extravagation of red blood cells. Inflammatory cells (i.e. neutrophils) are among the micro-haemorrhage. (e) Ventriculitis is persistent at 6 days post-inoculation and ventriculomegaly ensues with massive dilation of the ventricles. (f) Normal neuron (open arrow) and morphological features of neuronal programmed cell death demonstrated by hyperchromatic, condensed DNA in the cortex (solid arrows) at day 5. (g) Inflammatory foci in close proximity to brain capillaries at day 5. (h) Marginated neutrophils and infiltrating inflammatory cells at day 7. (i) Marginated neutrophils and inflammatory foci (arrow) at day 7. (j) Marginated neutrophils, leptomeningitis dissemination and dilated perivascular space. (k) Micro-abscess (arrow) (I) Marginated neutrophils in an abnormal blood vessel and inflammatory foci at day 9. 
bacteria and inflammatory cells into the brain, is compromised. During meningitic infections lesions frequently occur where the Virchow-Robin (perivascular) space fuses with the cortex. In this study the VirchowRobin space was dilated and contained inflammatory cells such as macrophages, sensing lymphocytes and local inflammatory cells that can recirculate as antigen-presenting cells (Fig. 1j). Multiple inflammatory foci within the brain closely associated with blood vessels suggest that in this model bacteria travel down the perivascular spaces (Fig. 1). This suggests a way that the bacteria may enter the CSF, causing a massive influx of inflammatory cells into the ventricles and meninges that may break down adhesion junctions and give access to the brain parenchyma. Microabscesses, inflammatory foci and marginated neutrophils were visible at day 9 (Fig. 1k, l). Electron microscopy was used to further discern the host cell interactions and intracellular location of internalized bacteria. Electron microscopic analysis of infant rat brains 7 days postinoculation found Ent. sakazakii in spacious membranebound phagosomes within infiltrating neutrophils and macrophages associated with inflammation (Fig. 2).

\section{Rat brain capillary endothelial cell invasion by Ent. sakazakii}

The pathogenesis of Ent. sakazakii meningitis involves haematogenous spread to the CNS and penetration of the $\mathrm{BBB}$. The pathogenesis of most infant meningitis involves invasion of the BBB. This has long been assumed of Ent. sakazakii meningitis due to a lack of any other biologically plausible explanation. In order to demonstrate BBB invasion the gentamicin protection assay was performed using the rat rBCEC4 cell line. C. koseri, C. freundii and E. coli $\mathrm{K} 1$ are reported to invade human microvascular endothelial cells and were used as positive controls. Ent. sakazakii strains NTU2, NTU658 and NTU57 had average percentage invasion values ranging from 0.43 to $1 \%$, which were not significantly different $(P=0.095,0.552$ and 0.672 , respectively) from positive control $E$. coli K1
$(0.57 \pm 0.08 \%)$ or C. freundii $(0.54 \pm 0.09 \%$; Fig. 3$)$. The C. koseri positive control was extremely invasive $(2.95 \%)$ while E. coli K-12 did not invade this cell line (Fig. 3). Strains NTU1, NTU3 and NTU84 were at least twofold less invasive than $E$. coli $\mathrm{K} 1(P \leqslant 0.0002)$ in vitro (Fig. 3$)$ and this has been shown to be biologically relevant since $0.1 \%$ invasion is associated with enhanced CNS entry in vivo (Badger \& Kim, 1998; Wang et al., 1999; Badger et al., 2000). Strains NTU1, NTU3 and NTU84 were also significantly less invasive than NTU2 $(P=0.001,0.041$ and 0.002 , respectively) in vitro (Fig. 3), further illustrating that invasiveness was dependent on strain. Our study appears to be the first to show that Ent. sakazakii invades capillary endothelial cells, suggesting that some strains from clusters 1 and 2 may be more likely to invade the BBB and cause CNS infection.

\section{Ent. sakazakii persists within human macrophages}

Macrophage uptake of invading microbes is an innate process that employs antimicrobial defence mechanisms such as oxidative burst, acidic compartmentalization and nutrient deprivation to eliminate harmful pathogens. The ability of some bacteria to persist or replicate within these long-lived immune cells offers protection from the immune response. Human U937 macrophages were utilized to determine if Ent. sakazakii was able to persist within human macrophages following phagocytosis. All strains of Ent. sakazakii tested were able to persist in macrophages up to $96 \mathrm{~h}$. However, this ability differed between strains. Strain NTU2 was able to replicate significantly over this time period while strains NTU1, NTU57 and NTU84 showed modest levels of replication (Fig. 4). Strain NTU3 (16S rDNA cluster group 3) showed the least resistance to macrophage killing among Ent. sakazakii strains (Fig. 4). E. coli K1 (persists), E. coli K-12 (killed), and C. koseri strain 319 (replicates) were used as controls.

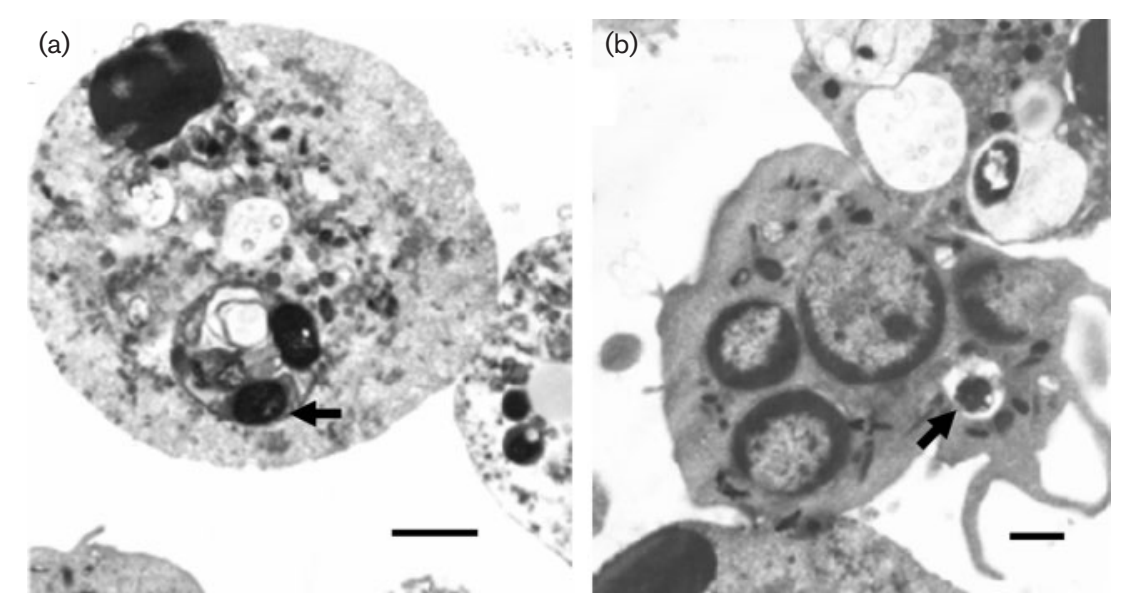

Fig. 2. Transmission electron micrographs of Ent. sakazakii in the infant rat brain. (a) Ent. sakazakii NTU57 (arrowed) was found in neutrophils (scale bar, $2 \mu \mathrm{m}$ ) and (b) membrane-bound vacuoles of macrophages (arrowed) associated with chronic-pattern inflammation within the brain at 6 days postinoculation (scale bar, $1 \mu \mathrm{m}$ ). 


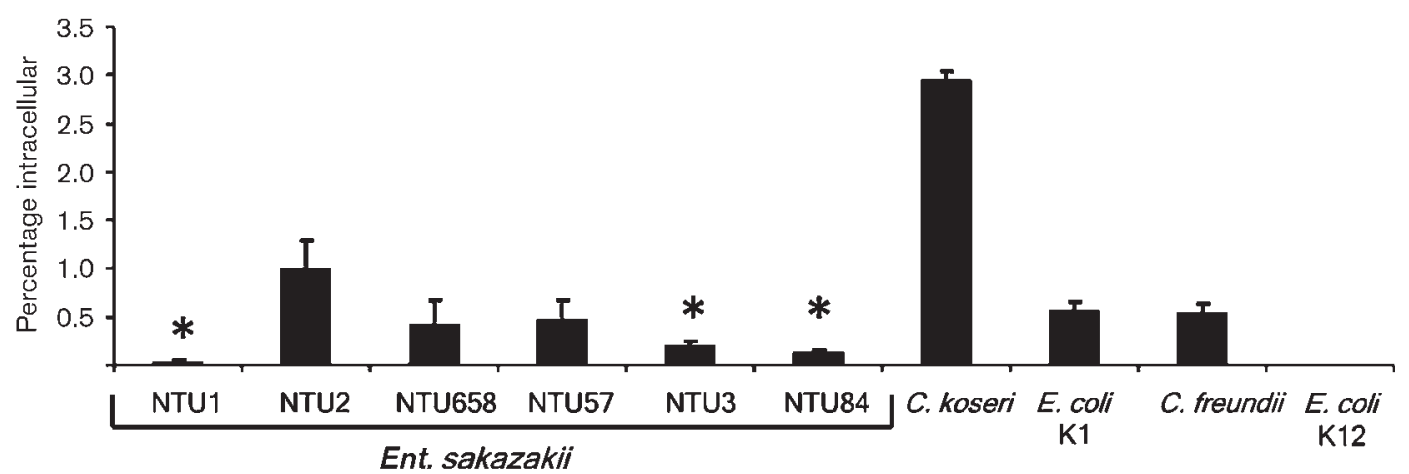

Fig. 3. Ent. sakazakii invasion of rat brain capillary endothelial cells. The gentamicin protection assay was performed using rat rBCEC4 cells to model the BBB invasion. Mid-exponential-phase bacteria were inoculated and incubated for $1.5 \mathrm{~h}$ then treated with gentamicin for $30 \mathrm{~min}$. No bacteria were recovered at $t_{0}$. Results for $t_{2}$ are presented as the percentage of the initial inoculum that was intracellular after 2 h. E. coli K-12 (non-invasive), C. koseri strain SMT319, C. freundii strain 340 and E. coli K1 (invasive) were used as controls. An asterisk marks the means with $P$ values $(P \leqslant 0.041)$ significantly less invasive than Ent. sakazakii NTU2, C. freundii strain 340 and E. coli K1. Data are means \pm SEM from two independent assays performed in triplicate.

\section{Comparative resistance to serum, acid and reactive oxygen}

The innate immune system facilitates a quick, non-specific immune response. Neonate susceptibility may be increased due to reduced gastric acid secretion up to $5 \mathrm{~h}$ immediately following birth (Euler et al., 1977). Bacteria that gain access to the bloodstream will be exposed to complement factors that cause cell lysis. Polymorphonuclear leukocytes are capable of releasing reactive oxygen species that damage cell membranes, proteins and essential macromolecules such as DNA. Ent. sakazakii strains were tested to determine resistance to acid, complement and reactive oxygen species. Cluster 3 and 4 strains and NTU1 were not resistant to serum complement (data not shown). All strains were resistant to acid at $\mathrm{pH} 4$ except NTU1. Superoxide dismutases (SODs) are metalloenzymes which are found in all aerobic organisms and help protect bacteria from oxidative stress. All bacterial strains tested showed some level of SOD activity between 0.23 and 2.0 units $\mathrm{ml}^{-1}$ as assessed by the SOD assay (Fig. 5). Ent. sakazakii strains NTU1, NTU2, NTU3 and NTU84 demonstrated the highest SOD activity. Among Ent. sakazakii strains, NTU1 and NTU84 had significantly higher activity than NTU658 $(P \leqslant 0.02)$ and NTU57 $(P \leqslant 0.03)$. All the Ent. sakazakii strains exhibited SOD activity significantly lower than the positive control value $(P \leqslant 0.05)$ that has a demonstrable affect on Y. entercolitica virulence (Roggenkamp et al., 1997); however, these studies demonstrate that SOD is functioning in Ent. sakazakii. While SOD is generally stationary-phase induced, and Fe-SOD is constitutively expressed, further sodA (Mn-SOD) activity may rely on specific induction such as acidic conditions that lead to oxidative stress (Kim et al., 2005). Deinococcus radiodurans is an example of a bacterium that can survive long term under harsh conditions, a property attributed to its strong SOD and DNA-repair activity (Tian et al., 2004). Ent. sakazakii is also known to

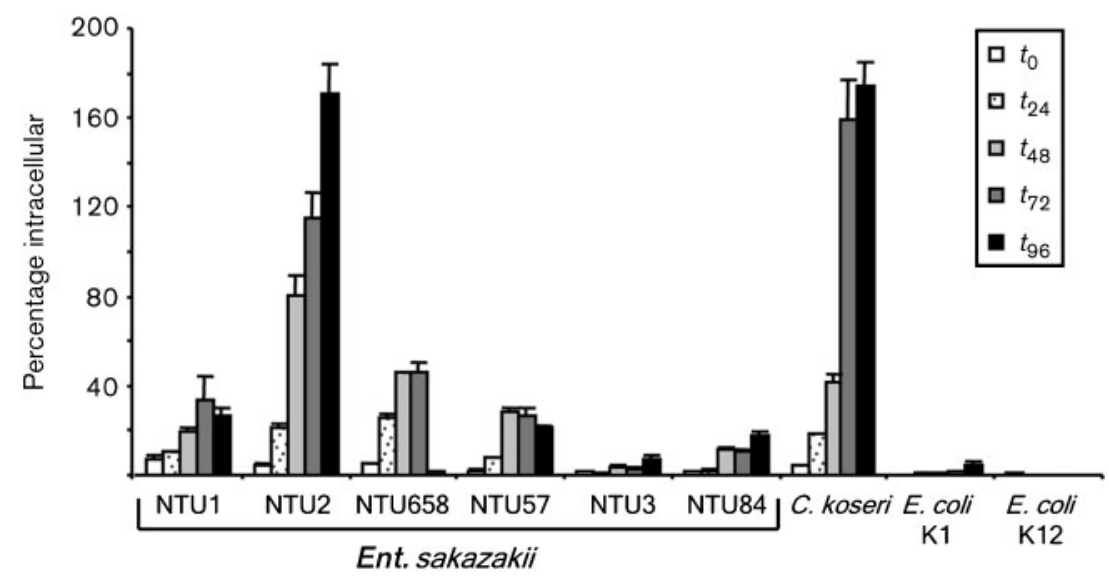

Fig. 4. Long-term survival of Ent. sakazakii within macrophages. The gentamicin protection assay was performed on human U937 macrophages using mid-exponential-phase bacteria at $24 \mathrm{~h}$ time points up to $96 \mathrm{~h}\left(t_{96}\right)$. Results are presented as the percentage of the initial inoculum that was intracellular. Data are means \pm SEM from a representative assay performed in triplicate. E. coli $\mathrm{K} 1$ (persists), E. coli K-12 (killed), and C. koseri strain SMT319 (replicates) were used as controls. 


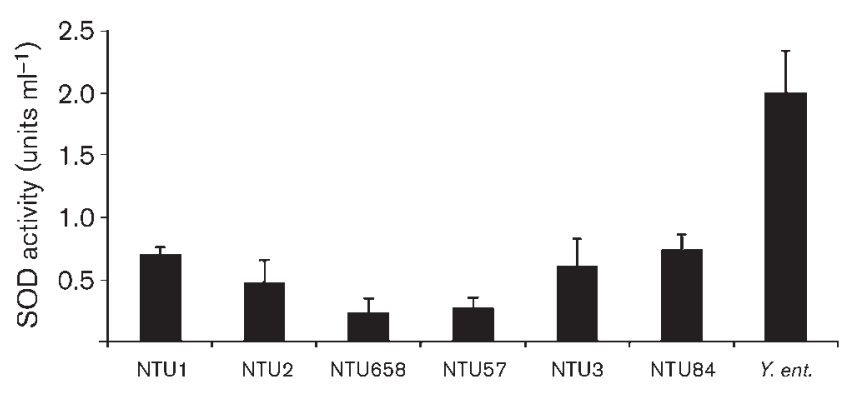

Fig. 5. Ent. sakazakii SOD activity. Supernatants from sonicated bacterial pellets were assessed for SOD activity. The range of SOD detection was between 0.025 and 0.25 units SOD $\mathrm{ml}^{-1}$. One unit of SOD is the amount of enzyme resulting in $50 \%$ dismutation of the superoxide radical. $Y$. enterocolitica was used as a positive control. Data are means \pm SEM from two independent assays performed in duplicate.

survive long term in powdered infant formula (CaubillaBarron \& Forsythe, 2007). It is possible that SOD activity may contribute to this ability; this is currently under investigation.

\section{Gene probes for ompA and sodA}

The ompA and sodA genes were identified in a DNA-DNA microarray analysis showing hybridization of Ent. sakazakii genes to Salmonella ompA and sodA DNA probes. This was confirmed by recent studies (Mohan Nair \& Venkitanarayanan, 2006) and the finding of a partial gene sequence for $\operatorname{sod} A$ and $\operatorname{sod} B$ in the ongoing genome sequencing project (http://www.genome.wustl.edu) using the BLASTX program with homologous Salmonella Typhimurium LT2 sequences. The $\operatorname{sod} A$ and $\operatorname{sod} B$ genes are known to protect bacteria from oxidative stress (McCord \& Fridovich, 1969) by catalysing the conversion of oxygen radicals to hydrogen peroxide (Farr \& Kogoma, 1991). Sensitivity to oxidative stress may contribute to reduced virulence so we considered the capability of Ent. sakazakii to survive early contact with the macrophage oxidative burst (Beaman \& Beaman, 1984) in addition to long-term persistence. While sodA was amplified from Ent. sakazakii cluster groups 1,2 and 4 and C. koseri, it was not from cluster group 3 Ent. sakazakii strain NTU3 (data not shown). NTU3 was also the only strain found to be less numerous in macrophages at $t_{24}$ compared to $t_{0}$ (Fig. 4). However, the SOD assay results do not correspond to these data, in that NTU3 showed one of the highest SOD activity values (Fig. 5); this suggests that the poor ability of this strain both to survive early macrophage interactions and to replicate in macrophages may not be a result of a diminished SOD activity or lack of the sodA gene. The lack of amplification cannot rule out the presence of $\operatorname{sodA}$ in this strain, as it is a gene that exhibits a degree of variability. Considering the concern for rapid accurate identification of Ent. sakazakii it is of interest that the variability of sodA has recently been utilized in multiplex PCR reactions to identify enterococci at the genus and species level (Jackson et al., 2004). With respect to infection, inactivation of sodA in $Y$. enterocolitica resulted in a marked reduction in virulence of the organism in a mouse infection model (Roggenkamp et al., 1997). However, inactivation of sodA in Salmonella Typhimurium did not significantly attenuate infection in mice, which suggests that SodA is only required for initial resistance to macrophage oxidative burst. Studies utilizing deletion mutants are ongoing to clearly define the importance of sodA during Ent. sakazakii infection.

In E. coli $\mathrm{K} 1$ the ompA gene has been linked with serum resistance and CNS invasion in the neonatal rat model (Weiser \& Gotschlich, 1991). The ompA gene was not amplified from cluster 4 strain NTU84 (data not shown). Our studies also show that NTU84 is serum sensitive. Since OmpA is reported to contribute to serum resistance we sought to determine if the presence of ompA among cluster 4 strains was associated with serum sensitivity. However, all other cluster 4 strains had the ompA gene and these strains were serum sensitive (data not shown), suggesting that OmpA may not play an essential role in Ent. sakazakii serum resistance. OmpA has been associated with increased HBMEC invasion in vitro (Shin et al., 2005). NTU84 had relatively low invasive abilities in our endothelial cell line, suggesting that OmpA may influence capillary endothelial cell invasion. Alternatively, analogous mechanisms for resistance and invasion may not be present in NTU84. The development and specificity of the ompA PCR as a potential tool for the rapid detection of Ent. sakazakii in infant formula is impressive (Mohan Nair \& Venkitanarayanan, 2006). However, the finding of an Ent. sakazakii strain (NTU84) that is not amplified with this method suggests that ompA PCR would not detect Ent. sakazakii in every case.

\section{Cytokine secretion from U937 macrophages containing Ent. sakazakii}

Since macrophages are thought to be early regulators of the innate immune response that further dictates the adaptive immune response, cytokine secretion from macrophages was assessed via ELISA following Ent. sakazakii inoculation. TNF $\alpha$ levels were not significantly different from 6 to $24 \mathrm{~h}$ and strain NTU84 was most robust, secreting $500 \mathrm{pg} \mathrm{TNF} \alpha \mathrm{ml}^{-1}$ after $24 \mathrm{~h}$ (Fig. 6a). IL-6 levels more than doubled and again strain NTU84 had the most robust response, secreting nearly $1200 \mathrm{pg} \mathrm{IL}-6 \mathrm{ml}^{-1}$, indicative of a strong inflammatory response elicited from macrophages in response to NTU84 infection (Fig. 6b). Both patterns of cytokine expression match those expected: TNF $\alpha$ expression occurs rapidly and levels out while IL-6 gradually increases over time. IL-10 secretion was induced (Fig. 6c); however, IL-12 was not recovered in significant amounts (data not shown). Cytokine levels in serum collected from 

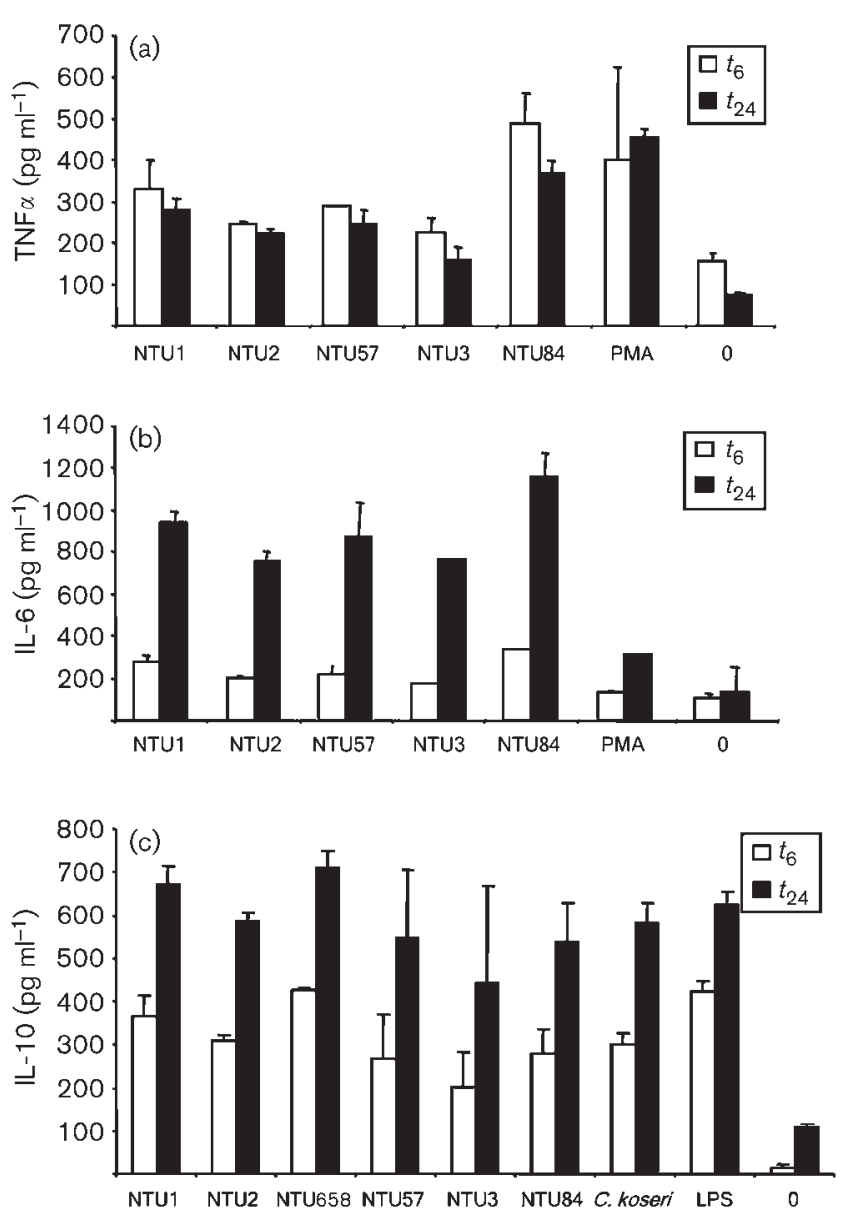

Fig. 6. Cytokine secretion from Ent. sakazakii-exposed U937 macrophages. Human U937 macrophages were inoculated with Ent. sakazakii strains as in the gentamicin assay. Supernatant samples were collected after 6 and $24 \mathrm{~h}$ incubation ( $t_{6}$ and $t_{24}$ ). ELISA was used to determine the concentration of (a) TNF $\alpha$, (b) IL6, (pro-inflammatory cytokines), and (c) IL-10 (immunosuppressive cytokine). IL-12 was not recovered in significant amounts. Data are means \pm SEM from duplicate assays.

neonatal rats $24 \mathrm{~h}$ following i.p. inoculation with strain 2 were also tested using ELISA. The level of IL-10 elicited by strain 2 was $562 \pm 133 \mathrm{pg} \mathrm{ml}^{-1}$. The level of IL- 6 was $2199 \pm 497 \mathrm{pg} \mathrm{ml}^{-1}$. The type 2 immune response is induced when the IL-10/IL-12 ratio is high, because antiinflammatory IL-10 can suppress pro-inflammatory IL-12. This response is insufficient at clearing intracellular infections. An early bias towards a type 2 immune response may contribute to an inability to successfully eliminate this intracellular pathogen.

\section{Concluding remarks}

Ent. sakazakii is a heterogeneous bacterial species, containing strains with disparate phenotypic and genotypic characteristics that have yet to be clearly characterized. In this study we comparatively analysed Ent. sakazakii for a number of known virulence traits and studied the host immune response in an attempt to gain a broader understanding of the phenotypic and infective differences that occur at the strain level. During meningitis additional damage is almost certainly caused by the host response to the pathogen. While the host response serves a protective purpose, it can be indiscriminate and cause irreversible damage to host brain tissues. More studies are needed; however, modulation of the host response may become an important way to mitigate the long-term sequelae of Ent. sakazakii meningitis. In the short term, reducing the bacterial load via antibiotics helps lessen the morbidity and mortality of meningitis but it is best to avoid exposing neonates to these organisms altogether.

\section{ACKNOWLEDGEMENTS}

We would like to thank Michael McClelland and Steffen Porwollik for Salmonella microarrays, and the University of Washington St Louis for making raw sequence available online for Ent. sakazakii. We acknowledge and thank Ingolf E. Blasig for providing the rBCEC4 cell line and Hiroyuki Shimada for the EM images. We are grateful to Georgina Manning and Alan McNally for critical evaluation and helpful discussions. This work was generously supported by Children's Hospital Los Angeles Department of Pathology start-up funds (J. L. B.).

\section{REFERENCES}

Badger, J. L. \& Kim, K. S. (1998). Environmental growth conditions influence the ability of Escherichia coli $\mathrm{K} 1$ to invade brain microvascular endothelial cells and confer serum resistance. Infect Immun 66, 5692-5697.

Badger, J. L., Stins, M. F. \& Kim, K. S. (1999). Citrobacter freundii invades and replicates in human brain microvascular endothelial cells. Infect Immun 67, 4208-4215.

Badger, J. L., Wass, C. A., Weissman, S. J. \& Kim, K. S. (2000). Application of signature-tagged mutagenesis for identification of Escherichia coli $\mathrm{K} 1$ genes that contribute to invasion of human brain microvascular endothelial cells. Infect Immun 68, 5056-5061.

Beaman, L. \& Beaman, B. L. (1984). The role of oxygen and its derivatives in microbial pathogenesis and host defense. Annu Rev Microbiol 38, 27-48.

Biering, G., Karlsson, S., Clark, N. C., Jonsdottir, K. E., Ludvigsson, P. \& Steingrimsson, O. (1989). Three cases of neonatal meningitis caused by Enterobacter sakazakii in powdered milk. J Clin Microbiol 27, 2054-2056.

Blasig, I. E., Giese, H., Schroeter, M. L., Sporbert, A., Utepbergenov, D. I., Buchwalow, I. B., Neubert, K., Schonfelder, G., Freyer, D. \& other authors (2001). ${ }^{\star} \mathrm{NO}$ and oxyradical metabolism in new cell lines of rat brain capillary endothelial cells forming the blood-brain barrier. Microvasc Res 62, 114-127.

Caubilla-Barron, J. \& Forsythe, S. J. (2007). Persistence of desiccated Enterobacter sakazakii and other Enterobacteriaceae in infant milk formula over a two year period. J Food Prot (in press).

Coignard, B., Vaillant, V., Vincent, J.-P., Leflèche, A., MarianiKurkdjian, P., Bernet, C., L'Hériteau, F., Sénéchal, H., Grimont, P., Bingen, E. \& Desenclos, J.-C. (2006). Infections sévères à Enterobacter sakazakii chez des nouveau-nés ayant consommé une préparation en 
poudre pour nourrissons, France octobre-décembre 2004. BEH 2006 (2-3), 10-13.

Drudy, D., O'Rourke, M., Murphy, M., Mullane, N. R., O'Mahony, R., Kelly, L., Fischer, M., Sanjaq, S., Shannon, P. \& other authors (2006). Characterization of a collection of Enterobacter sakazakii isolates from environmental and food sources. Int J Food Microbiol 110, 127-134.

Euler, A. R., Byrne, W. J., Cousins, L. M., Ament, M. E. \& Walsh, J. H. (1977). Increased serum gastrin concentrations and gastric acid hyposecretion in the immediate newborn period. Gastroenterology 72, 1271-1273.

FAO-WHO (2006). Enterobacter sakazakii and Salmonella in powdered infant formula. Second Risk Assessment Workshop, WHO Rome, Italy.

Farr, S. B. \& Kogoma, T. (1991). Oxidative stress responses in Escherichia coli and Salmonella typhimurium. Microbiol Rev 55, 561585.

Himelright, I., Harris, E., Lorch, V. \& Anderson, M. (2002). Enterobacter sakazakii infections associated with the use of powdered infant formula - Tennessee, 2001. MMWR Morb Mortal Wkly Rep 51, 298-300.

Iversen, C. \& Forsythe, S. J. (2007). Comparison of media for the isolation of Enterobacter sakazakii. Appl Environ Microbiol 73, 48-52.

Iversen, C., Waddington, M., Farmer, J. J., III \& Forsythe, S. J. (2004). Identification and phylogeny of Enterobacter sakazakii relative to Enterobacter and Citrobacter species. J Clin Microbiol 42, 5368-5370.

Iversen, C., Lancashire, L., Waddington, M., Forsythe, S. \& Ball, G. (2006). Identification of Enterobacter sakazakii from closely related species: the use of artificial neural networks in the analysis of biochemical and 16S rDNA data. BMC Microbiol 6, 28-35.

Jackson, C. R., Fedorka-Cray, P. J. \& Barrett, J. B. (2004). Use of a genus- and species-specific multiplex PCR for identification of enterococci. J Clin Microbiol 42, 3558-3565.

Jarvis, C. (2005). Fatal Enterobacter sakazakii infection associated with powdered infant formula in a neonatal intensive care unit in New Zealand. Am J Infect Control 33, e19.

Kim, K. S., Wass, C. A. \& Cross, A. S. (1997). Blood-brain barrier permeability during the development of experimental bacterial meningitis in the rat. Exp Neurol 145, 253-257.

Kim, J. S., Sung, M. H., Kho, D. H. \& Lee, J. K. (2005). Induction of manganese-containing superoxide dismutase is required for acid tolerance in Vibrio vulnificus. J Bacteriol 187, 5984-5995.

Kline, M. W., Kaplan, S. L., Hawkins, E. P. \& Mason, E. O., Jr (1988). Pathogenesis of brain abscess formation in an infant rat model of Citrobacter diversus bacteremia and meningitis. J Infect Dis 157, 106-112.

McCord, J. M. \& Fridovich, I. (1969). Superoxide dismutase. An enzymic function for erythrocuprein (hemocuprein). J Biol Chem 244, 6049-6055.

Mohan Nair, M. K. \& Venkitanarayanan, K. S. (2006). Cloning and sequencing of the ompA gene of Enterobacter sakazakii and development of an ompA-targeted PCR for rapid detection of Enterobacter sakazakii in infant formula. Appl Environ Microbiol 72, 2539-2546.
Muytjens, H. L., Zanen, H. C., Sonderkamp, H. J., Kollee, L. A., Wachsmuth, I. K. \& Farmer, J. J., III (1983). Analysis of eight cases of neonatal meningitis and sepsis due to Enterobacter sakazakii. J Clin Microbiol 18, 115-120.

Pagotto, F. J., Nazarowec-White, M., Bidawid, S. \& Farber, J. M. (2003). Enterobacter sakazakii: infectivity and enterotoxin production in vitro and in vivo. J Food Prot 66, 370-375.

Pagotto, F., Farber, J. M. \& Lenati, R. (2007). Chapter 5: Mechanisms of pathogenicity of E. sakazakii. In Enterobacter sakazakii. Edited by J. M. Farber \& S. Forsythe. Washington, DC: American Society for Microbiology.

Porwollik, S., Frye, J., Florea, L., Blackmer, F. \& McClelland, M. (2003). A non-redundant microarray of genes for two related bacteria. Nucleic Acids Res 31, 1869-1876.

Roggenkamp, A., Bittner, T., Leitritz, L., Sing, A. \& Heesemann, J. (1997). Contribution of the Mn-cofactored superoxide dismutase (SodA) to the virulence of Yersinia enterocolitica serotype O8. Infect Immun 65, 4705-4710.

Shin, S., Lu, G., Cai, M. \& Kim, K. S. (2005). Escherichia coli outer membrane protein A adheres to human brain microvascular endothelial cells. Biochem Biophys Res Commun 330, 1199-1204.

Sundstrom, C. \& Nilsson, K. (1976). Establishment and characterization of a human histiocytic lymphoma cell line (U-937). Int J Cancer 17, 565-577.

Tian, B., Wu, Y., Sheng, D., Zheng, Z., Gao, G. \& Hua, Y. (2004). Chemiluminescence assay for reactive oxygen species scavenging activities and inhibition on oxidative damage of DNA in Deinococcus radiodurans. Luminescence 19, 78-84.

Townsend, S. M., Pollack, H. A., Gonzalez-Gomez, I., Shimada, H. \& Badger, J. L. (2003). Citrobacter koseri brain abscess in the neonatal rat: survival and replication within human and rat macrophages. Infect Immun 71, 5871-5880.

Townsend, S., Caubilla-Barron, J., Loc-Carrillo, C. \& Forsythe, S. (2007). The presence of endotoxin in powdered infant formula milk and the influence of endotoxin and Enterobacter sakazakii on bacterial translocation in the infant rat. Food Microbiol 24, 67-74.

van Acker, J., de Smet, F., Muyldermans, G., Bougatef, A., Naessens, A. \& Lauwers, S. (2001). Outbreak of necrotizing enterocolitis associated with Enterobacter sakazakii in powdered milk formula. J Clin Microbiol 39, 293-297.

Wang, Y., Huang, S. H., Wass, C. A., Stins, M. F. \& Kim, K. S. (1999). The gene locus yijP contributes to Escherichia coli K1 invasion of brain microvascular endothelial cells. Infect Immun 67, 4751-4756.

Weiser, J. N. \& Gotschlich, E. C. (1991). Outer membrane protein A (OmpA) contributes to serum resistance and pathogenicity of Escherichia coli K-1. Infect Immun 59, 2252-2258.

Zaidi, T. S., Fleiszig, S. M., Preston, M. J., Goldberg, J. B. \& Pier, G. B. (1996). Lipopolysaccharide outer core is a ligand for corneal cell binding and ingestion of Pseudomonas aeruginosa. Invest Ophthalmol Vis Sci 37, 976-986.

Edited by: D. L. Gally 